Article

\title{
Full Polymer Dielectric Elastomeric Actuators (DEA) Functionalised with Carbon Nanotubes and High-K Ceramics
}

\author{
Tilo Köckritz ${ }^{1, *}$, René Luther ${ }^{2}$, Georgi Paschew ${ }^{2}$, Irene Jansen ${ }^{1,3}$, Andreas Richter ${ }^{2, *}$, \\ Oliver Jost ${ }^{3}$, Andreas Schönecker ${ }^{4}$ and Eckhard Beyer ${ }^{1,3}$ \\ 1 Chair of Laser and Surface Technology, Technische Universität Dresden, 01069 Dresden, Germany \\ 2 Chair of Polymeric Microsystems, Technische Universität Dresden, 01069 Dresden, Germany; \\ reneluther1@gmail.com (R.L.); georgi.paschew@tu-dresden.de (G.P.) \\ 3 Fraunhofer-Institut für Werkstoff- und Strahltechnik (Fraunhofer IWS), Winterbergstraße 28, \\ 01277 Dresden, Germany; irene.jansen@iws.fraunhofer.de (I.J.); oliver.jost@iws.fraunhofer.de (O.J.); \\ eckhard.beyer@iws.fraunhofer.de (E.B.) \\ 4 Fraunhofer-Institut für Keramische Technologien und Systeme (Fraunhofer IKTS), Winterbergstraße 28, \\ 01277 Dresden, Germany; andreas.schoenecker@ikts.fraunhofer.de \\ * Correspondence: tilo.koeckritz@tu-dresden.de (T.K.); andreas.richter7@tu-dresden.de (A.R.); \\ Tel.: +49-351-83391-3182 (T.K.); +49-351-46336-336 (A.R.)
}

Academic Editor: Joost Lötters

Received: 6 July 2016; Accepted: 5 September 2016; Published: 23 September 2016

\begin{abstract}
Dielectric elastomer actuators (DEA) are special devices which have a simple working and construction principle and outstanding actuation properties. The DEAs consist of a combination of different materials for the dielectric and electrode layers. The combination of these layers causes incompatibilities in their interconnections. Dramatic differences in the mechanical properties and bad adhesion of the layers are the principal causes for the reduction of the actuation displacement and strong reduction of lifetime. Common DEAs achieve actuation displacements of $2 \%$ and a durability of some million cycles. The following investigations represent a new approach to solving the problems of common systems. The investigated DEA consists of only one basic raw polymer, which was modified according to the required demands of each layer. The basic raw polymer was modified with single-walled carbon nanotubes or high- $k$ ceramics, for example, lead magnesium niobate-lead titanate. The development of the full polymer DEA comprised the development of materials and technologies to realise a reproducible layer composition. It was proven that the full polymer actuator worked according to the theoretical rules. The investigated system achieved actuation displacements above $20 \%$ regarding thickness, outstanding interconnections at each layer without any failures, and durability above 3 million cycles without any indication of an impending malfunction.
\end{abstract}

Keywords: electroactive polymers; sensors; actuators; conductive polymers; technologies for polymeric microsystems; full polymer actuator; electromechanical characterization; polydimethylsiloxane

\section{Introduction}

The initiative taken by governments to reduce emissions of $\mathrm{CO}_{2}$ during the production process and for the whole lifetime of products increases the interest in new and unconventional solutions. Therefore, industrial companies are finding ways to reduce pollution emissions and save valuable energy. Dielectric elastomer actuators (DEA) represent a promising field of research. The first investigated DEA was published by Röntgen at 1880 and since 1992 DEAs have been back in the focus of researchers and industrial operators as part of further investigations [1-4]. DEAs are not only applicable as actuators but also as sensors or energy harvesters [5-14]. DEAs consist at least 
of three layers, whereby a flexible dielectric is covered on both sides with compliant electrodes [3]. The operating principle of a DEA is based on the electrostatic pressure $p_{\mathrm{el}}=\varepsilon_{0} \cdot \varepsilon_{\mathrm{r}} \cdot U^{2} \cdot z^{-2}$, which causes the displacement of the actuator $s_{z}=-\varepsilon_{0} \cdot \varepsilon_{\mathrm{r}} \cdot U^{2} \cdot Y^{-1} \cdot z^{-2}[3,4]$. The specific values of the past equations are the relative dielectric coefficient $\varepsilon_{r}$, thickness of the dielectric $z$, elastic modulus $Y$ of the dielectric layer, permittivity of vacuum $\varepsilon_{0}$ and driving voltage $U$. Figure 1 visualise the operating principle of DEAs.

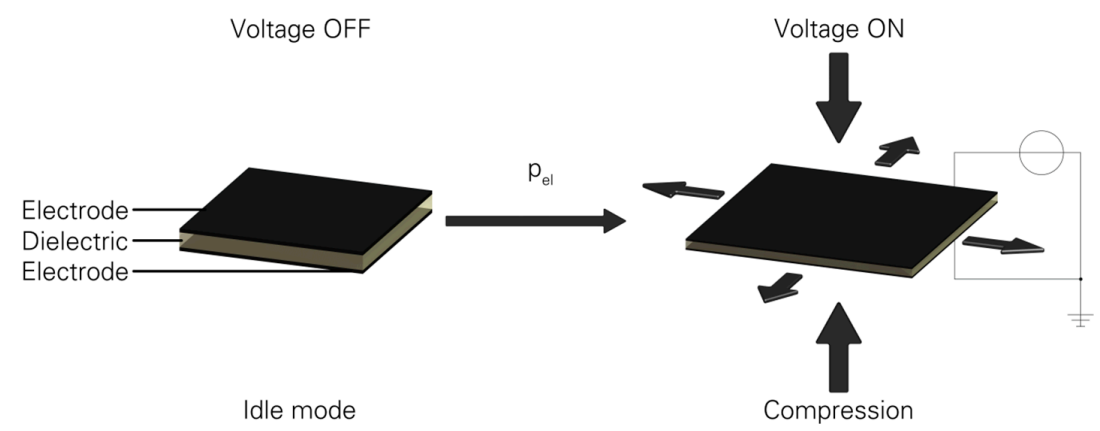

Figure 1. Scheme of the operating principle of a dielectric elastomer actuator (DEA) [3,4,15].

The main limiting factors of conventional DEAs are the achievable displacement and the durability. The layer composition is affected by significant differences regarding mechanical properties like elastic modulus and strain between the electrode and dielectric. Conventionally, electrodes consist of thin metallic layers, graphite grease, carbon black grease or carbon nanotubes (CNTs) [16-21]. The average durability for such DEAs with a dielectric layer consisting of polydimethylsiloxane (PDMS) is approximately $10^{8}$ cycles of actuation [16,20,22]. However, those electrodes consisting of grease containing graphite, silver and carbon achieve a higher durability like thin metallic electrodes $[16,20,22]$. Additionally, the adherence of the conventional electrodes to the dielectric and the brittleness of the electrodes are further limiting factors. Danfoss PolyPower ${ }^{\circledR}$ (Danfoss Polypower A/S, Nordborg, Denmark), which is the most common DEA material, reduces these problems by structuring the surface of the dielectric $[18,23]$. DEAs consisting of Danfoss PolyPower ${ }^{\circledR}$ with a back-to-back configuration achieves a maximum displacement of $2 \%$ with an applied field strength of $31.25 \mathrm{kV} / \mathrm{mm}$, which corresponds to a driving voltage of $2.5 \mathrm{kV}[18,24]$.

Furthermore, another approach to solve the problems of conventional DEAs is to build the whole actuator with only one basic raw material to achieve a full polymer DEA [25]. The required characteristics for dielectric and electrode layers are achieved by a modification of the basic raw polymer using respective fillers. Full polymer actuators will be able to address the incompatibilities of the DEAs, whereby durability and system performance will increase.

\section{Experimental Section}

\subsection{Materials}

The selection of the basic raw polymer is based on fundamental pre-investigations of two-components and additive curing PDMS. Three of the most important brands of PDMS were compared and analysed as a dielectric for DEAs. Here, the DowCorning Sylgard ${ }^{\circledR} 184$ (DowCorning Corp., Midland, MI, USA), Momentive RTV615 (Momentive Performance Materials GmbH, Leverkusen, Germany) and Wacker Elastosil ${ }^{\circledR}$ RT 625 (Wacker Chemie AG, München, Germany) were evaluated regarding their processability, reproducibility of the thickness and their mechanical parameters of the polymer layers. Further parameters like viscosity, pot-life and time for cross-linking were characterised. These factors are important for the modification and production of full polymer DEAs. The DowCorning Sylgard ${ }^{\circledR} 184$ satisfied the requirements at all points of interest and was chosen as basic raw polymer. DowCorning Sylgard ${ }^{\circledR} 184$ was purchased from Arrow Electronics Inc. (LS Venlo, 
The Netherlands) and Biesterfeld Spezialchemie GmbH (Hamburg, Germany) as kit [26]. The material used as electrical conductive filler were single-walled carbon nanotubes (SWCNTs) synthesised by Fraunhofer IWS, Dresden. The ferroelectric perovskite lead magnesium niobate-lead titanate (PMN-PT), which was used as high- $k$ dielectric filler, was synthesised by Fraunhofer IKTS, Dresden. Figure 2 shows scanning electron microscopy (SEM) images of the used (a) PMN-PT; and (b) SWCNTs. Additionally, common chemicals were applied during the whole investigation process. The preparation and cleaning of surfaces, devices and tools were done with ethanol (denatured), acetone, isopropanol and dichloromethane, which were obtained from Merck KGaA (Darmstadt, Germany).
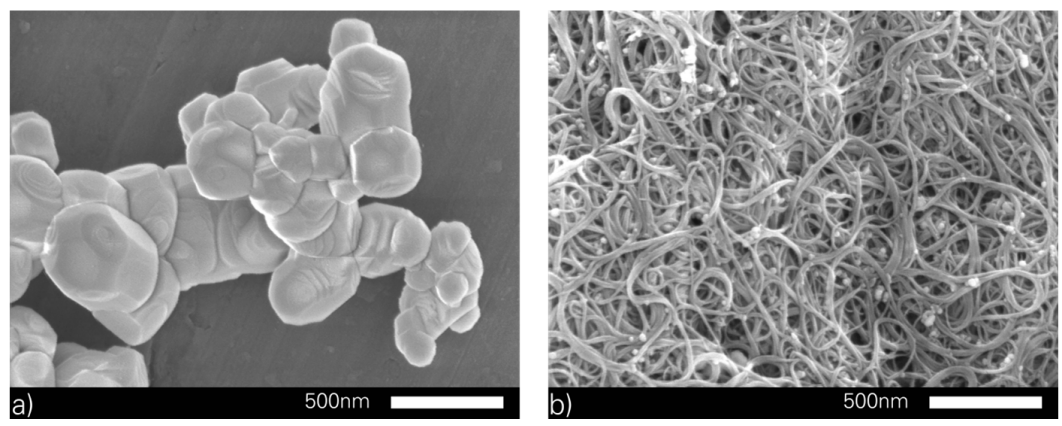

Figure 2. Scanning electron microscopy (SEM) image of (a) the perovskite lead magnesium niobate-lead titanate (PMN-PT) produced by Fraunhofer IKTS [15]; and (b) single-walled carbon nanotubes (SWCNTs) produced by Fraunhofer IWS [15].

\subsection{Synthesis of Single-Walled Carbon Nanotubes}

The SWCNTs were synthesised with a pulsed DC arc process. The process is based on a multi-component catalyser consisting of a mixture of cobalt, nickel, iron and molybdenum. The targets were vaporised, transported through the furnace by a gas flow and later on absorbed at a water-cooled collector. The values of the physical vapour deposition (PVD) process were a current of $100 \mathrm{~A}$, a voltage of $50 \mathrm{~V}$, a pulse-width of the pulsed DC arc at the range of dozens of ms, a furnace temperature of $1000{ }^{\circ} \mathrm{C}$ and a gas pressure of $100 \mathrm{mbar}$ [27].

The produced soot consists of $20 \mathrm{wt} \%$ of particles from the catalysts and $80 \mathrm{wt} \%$ of carbon. This carbon contains 40-60 wt \% of SWCNTs and these were divided into semiconducting and metallic SWCNTs. The content of the metallic SWCNTs was approximately $65 \mathrm{wt} \%$. Additionally, the soot was purified by a wet-chemical process with $\mathrm{HNO}_{3}$ and $\mathrm{H}_{2} \mathrm{O}_{2}$. The purification with $\mathrm{HNO}_{3}$ eliminates the metallic particles while the $\mathrm{H}_{2} \mathrm{O}_{2}$ eliminates the graphite. Figure 3 shows (a) the unpurified; and (b) purified SWCNT material. Finally, the purity of SWCNTs powder was approximately $98 \%$ of carbon content and only a few catalytic particles like nickel and cobalt. The SWCNTs show an average diameter of $1.25 \mathrm{~nm}$ [27-29].
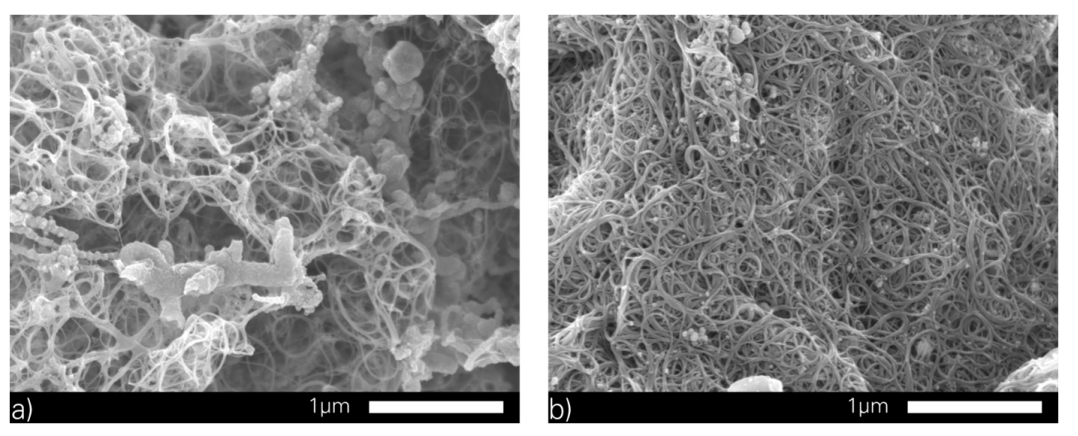

Figure 3. (a) SEM image of the produced material containing carbon, particles of the catalysts and SWCNTs; and (b) purified material. 


\subsection{Synthesis of Ferroelectric Perouskite PMN-PT}

The synthesised perovskite lead magnesium niobate (PMN), which can be assigned to the ferroelectric perovskites, will be stabilised with perovskite lead titanate (PT). Additionally, the PT decreases the curie-temperature to the range of room temperature. Thereby, PMN-PT exhibits the highest achievable permittivity at room temperature with approximately 30.00 and is well suited to high- $k$ ceramics. The composition of the used $\mathrm{PMN}-\mathrm{PT}$ was $0.67 \mathrm{~Pb}_{3} \mathrm{MgNb}_{2} \mathrm{O}_{9}-0.33 \mathrm{PbTiO}_{3}$. The resulting powder was only calcinated and not sintered [30-32].

\subsection{Design and Fabrication of the Elastomer Actuators}

\subsubsection{Design of the Elastomer Actuators}

The displacement of the actuator was characterised by using DEAs with a three layer composition and circular electrodes. Additionally, DEAs with a three layer composition and rectangular large-scale electrodes were used for investigating the durability. For such DEAs, the number of layers was increased up to 11 layers, in order to demonstrate the performance of full polymer multilayer DEAs as weightlifter and artificial muscle.

\subsubsection{Modification of the Basic Raw Material}

The modification of the basic raw polymer depends on the type of filler, morphology and kind of agglomeration. Figure 2 visualises the (a) PMN-PT; and (b) SWCNTs, which were used for the modification of the PDMS, and shows the differences regarding their morphology and agglomeration. The PMN-PT is nearly spherical, does not build strong agglomerates and needs only a homogenisation. Therefore, the integration was done by using a dual asymmetric centrifuge, produced by Hauschild Engineering \& Co. KG (Hamm, Germany). The SWCNTs are tube-like with a high aspect ratio, build strong agglomerates induced by their reactivity and need a deagglomeration and homogenisation. This was realised by a multistage process. At first, a homogenous premix was built by using the dual asymmetric centrifuge. Subsequently, the deagglomeration and homogenisation was done by a stepwise processing at a three roll mill, produced by EXAKT Advanced Technologies GmbH (Norderstedt, Germany) [33-36].

\subsubsection{Fabrication of Single Dielectric and Electrode Layers}

The investigations into the dielectric layer were based on two approaches. The first approach was to investigate the unmodified basic raw polymer, as the dielectric properties of the chosen PDMS are promising. The second approach was to investigate the modification of the basic raw polymer with PMN-PT to increase the actuation properties and to reduce the driving voltage. The filler content of the dispersions for the electrode layer was varied up to the highest amount of $3.0 \mathrm{wt} \%$ of SWCNTs to achieve the required electrical conductivity.

The fabrication of the layers began with the addition of the desired curing agent ratio to the different dispersions. This was mixed together by using the dual asymmetric centrifuge for $3 \mathrm{~min}$ at $3000 \mathrm{rpm}$. This material was used to applicate the film onto a carrier substrate. Previous investigations showed that float glass was a promising substrate because it has the lowest surface roughness and offered a sufficient anti-adhesion to the PDMS. The application was done with a COATMASTER 509 MC and a MULTCATOR 411, both produced by Erichsen GmbH \& Co. KG (Hemer, Germany), which is shown in Figure 4b. The thickness of each layer was adjustable between 0 and $1000 \mu \mathrm{m}$ by the coating knife. The width was limited to $150 \mathrm{~mm}$ and the length to $400 \mathrm{~mm}$.

\subsubsection{Fabrication of the Layer Composition of DEA}

Generally, the fabrication process of the different designs for DEAs was investigated for two different methods. The technological key factors of the investigation are the production of DEAs with thin dielectric and electrode layers without inhomogeneities and imperfections, a high variability 
of geometry and the opportunity to transfer, scale up and automate the technology. The main aim was to produce DEAs with a high reproducibility and durability, which enables long-term stability and outstanding actuation parameters. The fabrication was performed with the previously described methods for the production of single layers, since any variations of the fabrication process can influence the final material characteristics. Method 1 was based on previously produced and cross-linked electrode and dielectric layers, which were interconnected with an additional basic raw polymer adhesive layer. The major disadvantages of method 1 are the additional layer for the interconnection and a poorly heat-supported curing due to the different thermal expansion coefficients of the layers. In method 2, which is the most promising approach, the dielectric layers were previously produced and cross-linked and the electrodes were directly coated on the surface. The final shape of the electrode layer including the elastic and conductive paths was realised by masks. Multi-layer set-ups shall be stacked under wet or at not completely cross-linked conditions. Figure 4a visualises the fabrication process and (b) the used devices.

a)

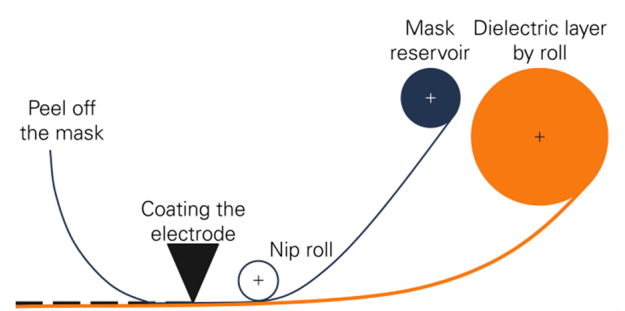

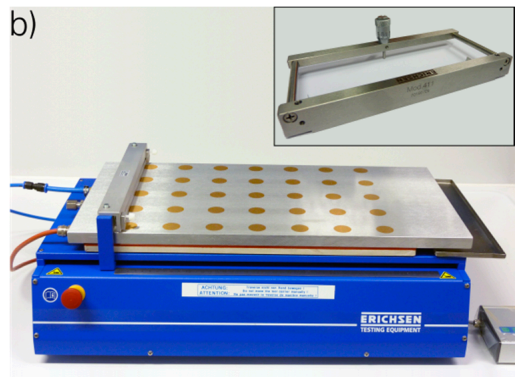

Figure 4. (a) Fabrication process for the DEA (method 2) [15]; and (b) the COATMASTER 509 MC and the MULTCATOR 411.

\subsection{Analytical Methods}

\subsubsection{Rheology Properties of Dispersions}

The rheological properties of the dispersion were investigated to evaluate the modification, type of fluid and processability of the dispersion modified with the nanoscale fillers. The main values used for the evaluation were the complex viscosity, storage and loss modulus related to the angular frequency. These factors may be used to determine the type of fluid and the rheological percolation threshold. The fluids are subdivided into Newtonian, shear-thickening or pseudoplastic character. As a consequence, the change in the processability is indicated by the rheological behaviour and can be adjusted to ensure a stable process. The rheology behaviour was measured by a digital rheometer C-VOR (Malvern Instruments GmbH, Herrenberg, Germany) [37,38].

\subsubsection{Electrical Properties of the Elastomer Films}

The dielectric properties were investigated by means of the electrical breakthrough for the dielectric with the investigated polymer electrodes and as comparison with sputtered electrodes of gold. The diameter of the electrodes was $2.0 \mathrm{~cm}$. The destructive testing was done with a HIPOT Tester with a maximum DC voltage of $12 \mathrm{kV}$, which was produced by Sefelec $\mathrm{GmbH}$ (Ottersweier, Germany) [39].

The electrical values were classified into two divisions with a threshold of $10^{5} \mathrm{Ohm} \cdot \mathrm{m}$, whereby the conductive and semi- and non-conductive materials were detached. The measurement above the threshold was done with an Electrometer/High Resistance Meter 6517B and Resistivity Test Fixture 8009, both produced by Keithley Instruments Inc. (Cleveland, OH, USA) [40-43]. The characterisation below the threshold was done with a Keithley Digital Multimeter 2000 and self-made fixture based on the four-wire-measurement [44-47]. The samples for the Resistivity Test Fixture 8009 are quadratic with a dimension of $6.5 \mathrm{~cm}$. The samples used for the four-wire-resistivity measurement are $7 \mathrm{~cm}$ long and $3 \mathrm{~cm}$ wide. Both were $140 \mathrm{~mm}$ thick. 


\subsubsection{Mechanical Properties of Films and Compounds}

The mechanical characterisation of the different layers was done to investigate the adjustment of the mechanical parameters. The test was used to investigate the elastic modulus and the shear tension together, which is based on the DIN EN ISO 527 norm [48,49] and ASTM D 638-14 [50]. The testing speed was $1 \mathrm{~mm} / \mathrm{min}$ at the elastic modulus and $10 \mathrm{~mm} / \mathrm{min}$ at the shear tension [48-50]. The used probes were $13.0 \mathrm{~cm}$ long, $2.5 \mathrm{~cm}$ wide and $0.04 \mathrm{~cm}$ thick.

The mechanical characterisation for the layer composition was done by peeling tests to examine the interconnection of the layers, which was based on DIN EN ISO 11339 [51] and ASTM D 1876-01 [52]. The testing speed was $10 \mathrm{~mm} / \mathrm{min}$, the probes were $13.0 \mathrm{~cm}$ long, $2.5 \mathrm{~cm}$ wide and $0.04 \mathrm{~cm}$ thick and the peeling distance was $6.0 \mathrm{~cm}[51,52]$. Both tests were done with the testing machine Zwick/Roell Z050 (Zwick GmbH \& Co. KG, Ulm, Germany) and the shear tension test was supported by the optical measurement system ARAMIS 5M (version 6.3, Gesellschaft für Optische Messtechnik mbH, Braunschweig, Germany) to characterise the real strain.

\subsubsection{Actuator Properties}

The characterisation of the displacement of the actuators is based on the principle of a mechanical thickness shear crystal, which was done for three layer actuators with circular electrodes [53]. Therefore, a high-precision experimental rig was designed, constructed and realised. The displacement of different actuators was determined by using an interferometric measuring system to evaluate the thickness variation of the DEA regarding driving voltage. In addition, an interferometer $\mathrm{ZMI}^{\mathrm{TM}} 7702$ by Zygo Corp. (Middlefield, CT, USA), a power supply HM8142 by Hameg Instruments GmbH (Germany) and a high-voltage amplifier 609B by Trek Inc. (Lockport, NY, USA) was used [54-56]. Additional information is shown in Section 1 of Supplementary Material.

The characterisation for large-scale DEAs was done according to the pure shear measurement described on [57] for the constant force method. This set-up was used to investigate the durability and displacement for three layer and multilayer DEAs as an artificial muscle acting as a weightlifter. The actuators were wound around two separate hollow cylinders and a tubeless DEA was built, which was only fixed by squeezing. The displacement was measured by elongation of the weight with the laser interferometer. Additional information is shown in Section 2 of Supplementary Material.

The investigations into lifetime were done for electro-mechanical stress induced by a determined driving voltage and a frequency. Additionally, the lifetime of the DEAs was investigated for mechanical stress induced by a determined elongation and a frequency, whereby a cyclic measurement of the electrical strength was executed.

\section{Results and Discussion}

\subsection{Fundamental Characterisation of the Basic Raw Polymer}

The mechanical properties like stress-strain behaviour and the elastic modulus-just as the dielectric strength of the unmodified basic raw polymers is a very important factor-can be controlled by changing the proportions of the curing agent ratio and the curing conditions. These factors are useable for the mechanical adjustment to achieve a correlation of the dielectric and electrode layers. On the other hand, it is possible to achieve reproducible parameters. These material properties of the silicone were investigated for a curing agent ratio of 8:1 up to 21:1. The recommended curing agent ratio for the PDMS was 10:1 [26]. The elastic modulus and stress-strain-behaviour can be regulated by a surplus or deficit of hardener. Hereby, a range of the elastic modulus between $2.72 \mathrm{MPa}(8: 1)$ and $0.52 \mathrm{MPa}(21: 1)$ was adjustable. The achievable strain was also adjustable between $80 \%$ and $170 \%$. The variation of the curing agent ratio does not influence the relative permittivity and an average value of 3.44 was investigated. The dielectric strength appeared to have an influence through its variation and the lowest dielectric strength was $78.8 \mathrm{kV} / \mathrm{mm}$. Figure $\mathrm{S} 2$ shows the investigated stress-strain behaviour, relative permittivity and dielectric strength corresponding to the curing agent ratio. 


\subsection{Rheology of the Dispersions}

The rheological properties of the basic raw polymer were affected by the modification with the nanoscale fillers. The change of the complex viscosity may become a crucial value for the material development regarding to the processability of the dispersions to monolithic full polymer DEAs. The morphology of the particles showed different behaviours regarding the affectation of the rheological behaviour, shown in Figure S3. The addition of $66.6 \mathrm{wt} \%$ of PMN-PT caused an increase in the complex viscosity from 4.5 Pas up to 9.4 Pas, whereby $3.0 \mathrm{wt} \%$ of SWCNTs reached $43.3 \mathrm{kPas}$, which can be explained by the aspect ratio. PMN-PT do not build strong interconnected networks in contrast to the SWCNTs. The dispersions containing PMN-PT showed a Newtonian behaviour and dispersions containing SWCNTs a shear thinning behaviour: in this case, a reduction of the complex viscosity may cause by an increased shear exposure. The rheological percolation threshold and change of the microstructure can be characterised by the storage or loss modulus for anisometric fillers, described by [38]. The investigations showed a percolation threshold of $0.5 \mathrm{wt} \%$ and a change of the microstructure. Figure S4 shows the investigated storage and loss modulus vs. the angular frequency. Additional information is presented in [33-36].

\subsection{Mechanical and Electrical Properties of the DEA}

\subsubsection{Electrode Layers}

The aim was to achieve the necessary electrical conductivity of the basic raw polymer by the integration of SWCNTs. The requirements to the electrical conductivity depend on the operating field of the further actuator. A high-dynamic operating of DEAs requires a high conductivity and under quasistatic conditions lower conductivities are required. The investigations of [58,59] showed that a sheet resistance significantly higher than $10 \mathrm{kOhmsq}$ is insufficient for the electrode layers for DEAs.

The modification of the basic raw polymer with SWCNTs had an influence on the mechanical values, which was negligible. The elastic modulus fluctuates in the range between $1.82 \mathrm{MPa}$ and 2.05 $\mathrm{MPa}$. The stress was reduced from $4.0 \mathrm{MPa}$ to $1.96 \mathrm{MPa}$ and the strain from $120 \%$ to $70 \%$ by the addition of $2.0 \mathrm{wt} \%$ of SWCNTs. Additionally, a counteracting effect should be possible through the adjustment of the basic raw polymer. Figure 5a shows the influence of the mechanical values.

The reduction of the specific resistivity achieved at the percolations threshold of $0.5 \mathrm{wt} \%$ of SWCNTs is nearly $90 \mathrm{Ohm} \cdot \mathrm{m}$ and for $3.0 \mathrm{wt} \% 1.7 \mathrm{Ohm} \cdot \mathrm{m}$, shown in Figure $5 \mathrm{~b}$. The basic raw polymer has a specific resistivity above $10^{14} \mathrm{Ohm} \cdot \mathrm{m}$. According to [58,59], the threshold was reached between $2 \mathrm{wt} \%$ and $3 \mathrm{wt} \%$, whereby $3.0 \mathrm{wt} \%$ scored $5.4 \mathrm{kOhm}$. Therefore, the filler content was determined to be $3.0 \mathrm{wt} \%$.

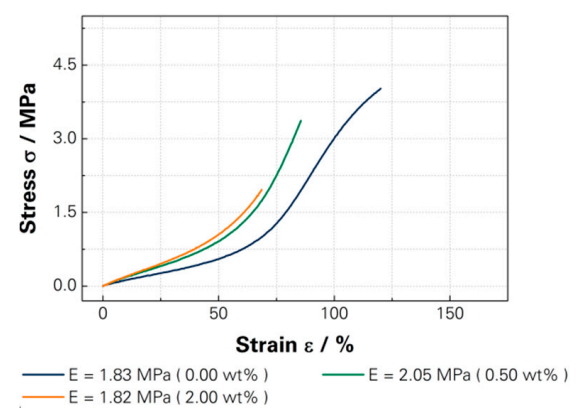

(a)

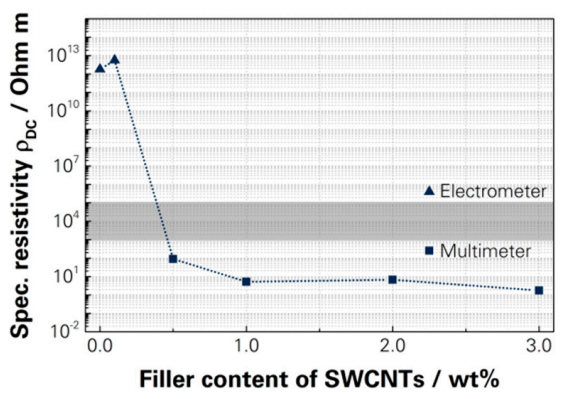

(b)

Figure 5. (a) Shift of the stress-strain behaviour [15]; and (b) the specific conductivity through the modification of SWNCTs. 


\subsubsection{Dielectric Layers}

The modification of the basic raw polymer with PMN-PT was focused on increasing the relative permittivity without having a strong influence on the other parameters like the elastic modulus, stress-strain behaviour or the complex viscosity. The first detected threshold was the processability of dispersions with a filler content beyond $50 \mathrm{wt} \%$. The particles accumulated in the coating knife gap and prevented the production of homogeneous and reproducible dielectric layers. Furthermore, the elastic modulus was significantly changed from 1.83 MPa for unfilled PDMS to 3.06 MPa for a filler content of $50 \mathrm{wt} \%$ of PMN-PT. The stress showed a slight reduction by the addition of $33.3 \mathrm{wt} \%$ of PMN-PT but again an increase to $3.8 \mathrm{MPa}$ for $50 \mathrm{wt} \%$ of PMN-PT. The strain was reduced from $120 \%$ to $80 \%$. If necessary, further adjustments of the mechanical parameters are possible. The relative permittivity was increased from 3.2 to 4.6 by the addition of $33.3 \mathrm{wt} \%$ and to 6.8 with $50.0 \mathrm{wt} \%$. The dielectric strength was for each step of modification above $80 \mathrm{kV} / \mathrm{mm}$. The plots are shown in Figure S5.

\subsubsection{Comparison of Full Polymer and Metallic Electrodes for DEAs}

The production process of the DEAs with sputtered electrodes is completely different to the fabrication of the full polymer DEAs. Therefore, the influence of the dielectric parameters was investigated for unmodified dielectrics and for dielectrics modified with PMN-PT. The dielectric benefits stemming from the polymer electrodes and a higher dielectric strength can be observed, which was leastwise $30 \mathrm{kV} / \mathrm{mm}$ higher compared to the values achieved with gold electrodes. The process for the deposition of the gold electrode seems to influence the properties of the dielectric layer and the vaporised gold may penetrate the surface, whereby the distance between the electrodes is reduced. Oppositely, the relative permittivity is not shown to have such a significant influence on the values. The investigated difference can be led back to variations of the thickness of the dielectric. Additionally, the influence of different curing agent ratios was also investigated and showed that the polymer electrodes achieve a higher dielectric strength than the sputtered electrodes by at least $40 \mathrm{kV} / \mathrm{mm}$. The variation from 10:1 to 16:1 caused an increase of the dielectric strength from $46.8 \mathrm{kV} / \mathrm{mm}$ to $51.14 \mathrm{kV} / \mathrm{mm}$ using sputtered electrodes and with polymer electrodes from $84.6 \mathrm{kV} / \mathrm{mm}$ to $107.4 \mathrm{kV} / \mathrm{mm}$. In comparison, the full polymer DEAs achieved distinctly higher dielectric strengths than metallic electrodes and Danfoss PolyPower ${ }^{\circledR}$. The plots are shown in Figures S6-S8.

\subsubsection{Three-Layer DEA}

The investigated production technology for the full polymer DEAs was construed as variable and scalable process. The investigated production technology satisfied a high variability of electrode geometries up to thin elastic conductive paths for the electrical connection of the DEAs, which is only restricted by the mask and structuring technology. The produced DEAs exhibit a high reproducibility with variations below 5\% regarding to the layer thickness and mechanical properties. Additionally, such DEAs achieved an outstanding stability of the different interconnected layers, which was proven by peel tests and SEM images. The peeling is the worst kind of exposure to the interconnection and peeling forces of $0.4 \mathrm{~N} / \mathrm{cm}$ were achieved. Additionally, the SEM image of cryogenic-fractured DEAs does not show any trapped air, kissing bonds or some other failures of the interconnection. Therefore, an outstanding durability of the DEAs was achieved and malfunctions caused by the production process and layer interconnection are unexpected. Figure 6a shows the peeling test and (b) a SEM image of a cryogenic-fractured three layer DEA. Figure $6 \mathrm{~b}$ visualises also a slight re-agglomeration of the SWCNTs along the fracture. The reactivity of the SWCNTs caused the re-agglomeration, which is positive for the electrical conductivity [60,61]. These sub-agglomerates build interconnections between the SWCNTs and reduce the percolation threshold [60,61]. Figure S9 shows three kinds of produced DEAs. 

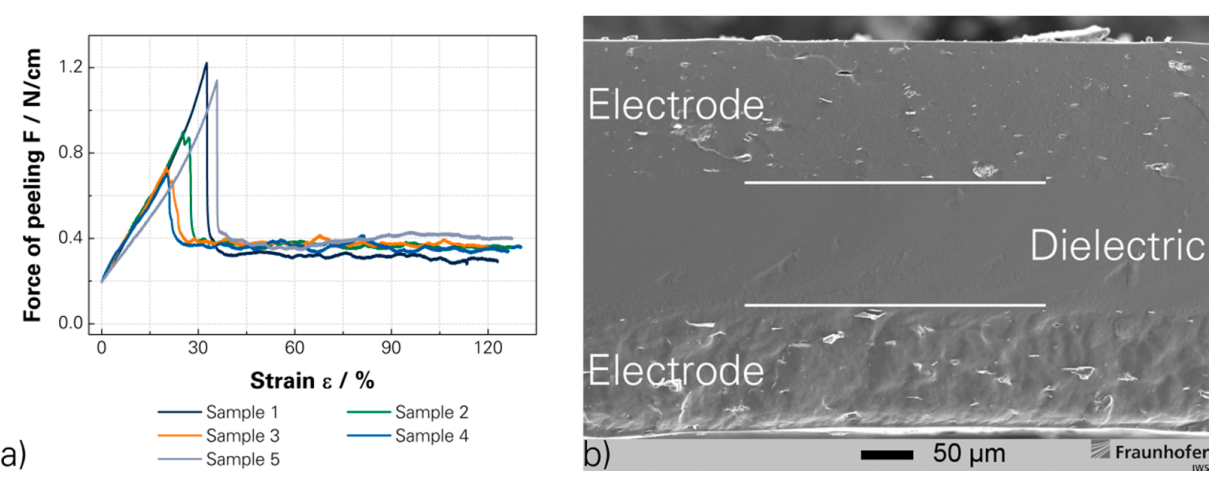

Figure 6. (a) Mechanical testing of the peeling force [15]; and (b) a cryogenic-fractured SEM image of a three layer DEA [15].

\subsection{Actuator Properties}

\subsubsection{Influence of Material and Design Properties}

The actuator properties were investigated mainly with DEAs produced by unmodified silicone as the dielectric layer and by $3 \mathrm{wt} \%$ SWCNTs modified basic raw polymer as the electrode material. The displacement $s_{z}$ of the DEAs can be influenced mainly by the relative permittivity, the elastic modulus and the thickness of the dielectric layer. The relative permittivity of the dielectric was adjustable by the addition of PMN-PT, the elastic modulus by the variation of the curing agent ratio or modification with PMN-PT, and the thickness of the dielectric layer can be reduced by the adjustment of the coating knife or further adaptions of the investigated production technology.

\subsubsection{Operation Parameters}

The investigation of the actuation displacement was done with predetermined operation parameters for driving voltage and times for charging, maximum load, discharging and without load. The displacement was investigated by a short term exposure with several repetitions. The specified times were exactly the same for each driving voltage and only the maximum load was changed. The time for charging and discharging was determined to be $10 \mathrm{~s}$ and the time under maximum load was $1 \mathrm{~s}$. Additionally, the maximum displacement for static loadings can be investigated. Here, the time under load was extended to $600 \mathrm{~s}$, which can be observed as static load. The determined regimes are presented in Figures S10 and S11.

\subsubsection{Actuation Behaviour}

The actuation behaviour was investigated for different configurations of three layer actuators to determine the influence of the curing agent ratio and the modification with PMN-PT of the dielectric layer, different kinds of operation parameters and the durability of the DEAs. The standard configuration of the actuators has an unfilled dielectric with a curing agent ratio of 10:1 and a geometry of $(15 \times 15 \times 0.011) \mathrm{cm}$, just as electrodes containing $3.0 \mathrm{wt} \%$ of SWCNTs with a diameter of $2 \mathrm{~cm}$. Table $\mathrm{S} 1$ gives a detailed overview of the actuators used and their configurations.

At first, the reaction time for the standard actuator was investigated for a driving voltage of $9000 \mathrm{~V}$ and reached $80 \mathrm{~ms}$, shown at Figure 7. The investigation was limited by the equipment and a further reduction should be possible. Additionally, Figure 7 shows that the main part of the displacement was reached directly after turning on even though a further displacement takes place during the subsequent period. This can be explained through the Mullins effect, whereby the elasticity of the polymer depends on the duration and count of cycles of the acting exposure [62-64]. Therefore, the actuation displacement achieved for the short time exposure can differ from a static exposure. The evaluation of short term exposure is done based on the measured displacements regarding the 
driving voltage for several cycles, which is used for calculating the average displacement. The average displacement of the driving voltages was used to calculate the characteristic curve of the actuator, as presented in Figure S12.

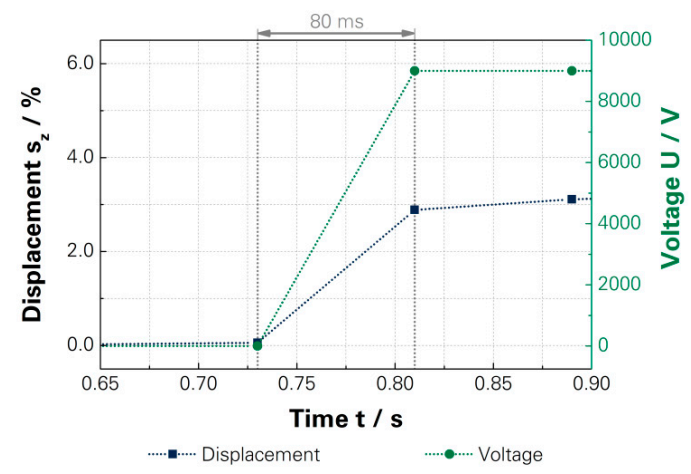

Figure 7. Investigated reaction time of a standard DEA [15]—-Table S1: actuator 1.

The investigation into the influence of the elastic modulus on the actuation displacement involved the examination of four values, adjusted by the variation of the curing agent ratio. The used DEAs had an elastic modulus of $1.83 \mathrm{MPa}(10: 1), 0.86 \mathrm{MPa}(13: 1), 0.81 \mathrm{MPa}(16: 1)$ and $0.51 \mathrm{MPa}(21: 1)$. The dependency of the actuation displacement was proven but the restoring forces significantly limited the increase of the displacement. The reduction of the elastic modulus to $0.81 \mathrm{MPa}$ caused an increase of the displacement but a further reduction of the elastic modulus caused a decrease of the displacement. The DEA with 0.81 MPa still achieved a displacement beyond the standard actuator and the DEA with $0.51 \mathrm{MPa}$ reached the lowest displacements. The DEAs achieved, with a driving voltage of $7000 \mathrm{~V}$, a displacement of 5.7\% (1.83 MPa), 13.3\% (0.86 MPa), 5.5\% (0.81 MPa) and 4.3\% (0.51 MPa). Figure 8 shows the measured actuation displacements and calculated characteristic curves for comparison, and the associated investigations are detailed in Figures S13-S16.

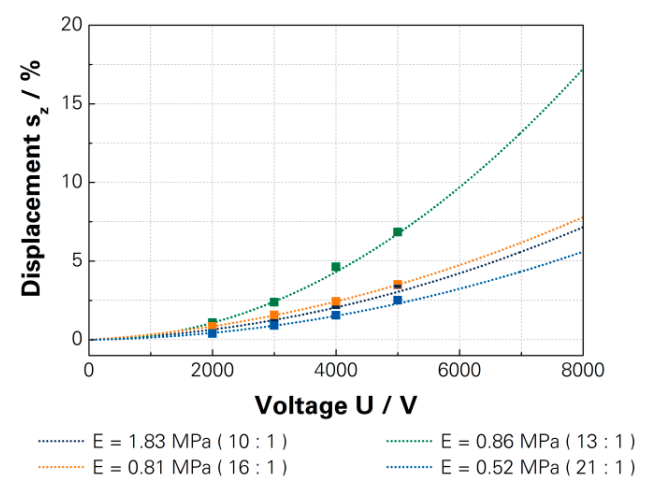

Figure 8. Measured actuation displacements and characteristic curves of full polymer DEA with different elastic modulus [15]—Table S1: actuators 2-5.

Additionally, the maximum displacement for the DEAs with an elastic modulus of $1.83 \mathrm{MPa}(10: 1)$ and $0.86 \mathrm{MPa}(13: 1)$ was investigated to represent the difference between the short term actuation and the maximum displacement. Likewise, the maximum displacements showed that the elastic modulus had a strong influence on the displacement. The DEAs achieved, with a driving voltage of $7000 \mathrm{~V}$, a maximum displacement of 5.3\% (1.83 MPa) and 19.1\% (0.86 MPa). The stiffer DEA does not show a significant difference between the short term actuation and the maximum displacement, while the elastic DEA showed a dramatic difference of $5.9 \%$. This can be explained by the Mullins effect, whereby the softer DEA allowed a greater elastic flow of the material depending on the duration of the acting 
exposure. Figure S17 shows the maximum displacement regarding the driving voltage for the stiff DEA (a); and the soft DEA (b).

The investigation into the influence of thickness on the actuation displacement of the dielectric layer was investigated for $46 \mu \mathrm{m}$ and $105 \mu \mathrm{m}$. The achieved actuation displacement showed a significant difference between the two DEAs, as shown in Figure 9. The reduction of the thickness can influence the electrical breakthrough, which is why the driving voltage has to be reduced to $3000 \mathrm{~V}$. The determined driving voltage corresponds to an electric field of $71 \mathrm{kV} / \mathrm{mm}$, which is still below the investigated dielectric strength for polymer electrodes of $84.6 \mathrm{kV} / \mathrm{mm}$, and prevents the destruction of the actuator. Therefore, the actuation displacement of the DEA with $105 \mu \mathrm{m}$ achieved $1.3 \%$ and the DEA with $46 \mu \mathrm{m}$ achieved $8.9 \%$. The actuation displacement for a driving voltage of $5000 \mathrm{~V}$ achieved $3.0 \%$ for the DEA with $105 \mu \mathrm{m}$ thickness and $23.1 \%$ for the thinner DEA, based on extrapolation of the calculated characteristic curves, which are shown in Figures S18 and S19.

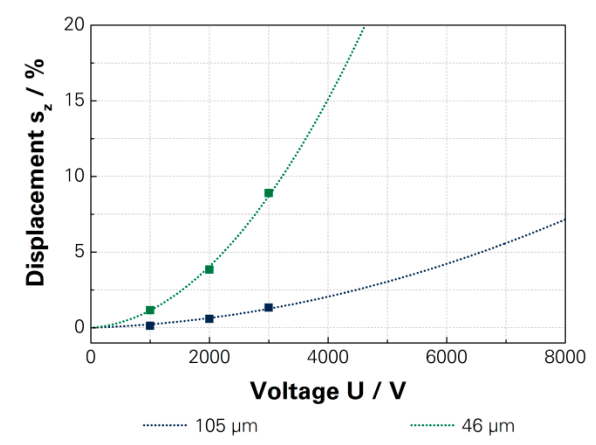

Figure 9. Measured actuation displacements and characteristic curves of full polymer DEA with different thick dielectric layers [15]—Table S1: actuators 2 and 7.

The influence of the relative permittivity on the actuation displacement was investigated for the PMN-PT modified basic raw polymer. The modification of the PDMS with the PMN-PT increased the relative permittivity but also the elastic modulus. The investigated DEAs achieved a relative permittivity of 3.24 (unmodified), 4.62 (33.3 wt \%) and $6.79(50.0 \mathrm{wt} \%)$. The main problem was that the elastic modulus increased faster than the relative permittivity by modification. Therefore, the actuation displacement was reduced. An additional counteracting effect from softening the dielectric layer was not investigated because the presented results based on softened DEAs with a curing agent ratio of 13:1. The achieved displacement with a driving voltage of $7000 \mathrm{~V}$ was $13.2 \%$ (3.24), 8.2\% (4.62) and $3.8 \%(6.79)$, which is shown in Figure 10 and is based on the calculated characteristic curves, shown in Figures S20-S22.

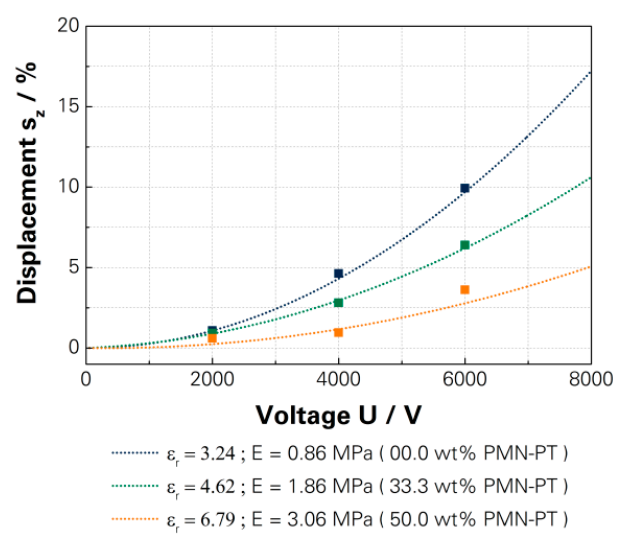

Figure 10. Measured actuation displacements and characteristic curves of full polymer DEA with different relative permittivities [15]—Table S1: actuators 2, 8 and 9. 
An important factor in the development process was the durability of the full polymer DEAs. The long-term-stability was proven in two ways. Firstly, the DEAs were stressed with $9 \%$ elongation and a repetition rate of $1 \mathrm{~Hz}$ by mechanical strain. The dielectrical strength was cyclically tested at a threshold of $66.6 \mathrm{kV} / \mathrm{mm}$. At one million cycles and beyond two million cycles, the tested dielectric strength was increased to $85 \mathrm{kV} / \mathrm{mm}$ to enhance the demands and the stress. The tests were proven successful for more than three million cycles. Secondly, the DEAs were stressed by electromechanical exposure as a weightlifter at a fixed driving voltage of $5000 \mathrm{~V}$, which corresponded to an electric field of $47.6 \mathrm{kV} / \mathrm{mm}$, and a repetition rate of $4 \mathrm{~Hz}$. The test was successfully driven for 140,000 cycles. The DEAs of both tests did not show any degradations or influences of the material and therefore further cycles will be achievable. Figure 11 showed the investigations for the (a) mechanical; and (b) electromechanical stressing. Additionally, a drift of the actuation displacement was investigated, which was also represented at the electromechanical stress, recognisable for the reduction in displacement every day. Associated investigations show a drift of the displacement, which was measurable up to 2700 cycles but the value of actuation displacement showed only a loss of $10 \%$. Beyond 2700 cycles, there was a displacement of $7.8 \%$ with a deviation of $0.07 \%$. Figure S23 shows the curves.
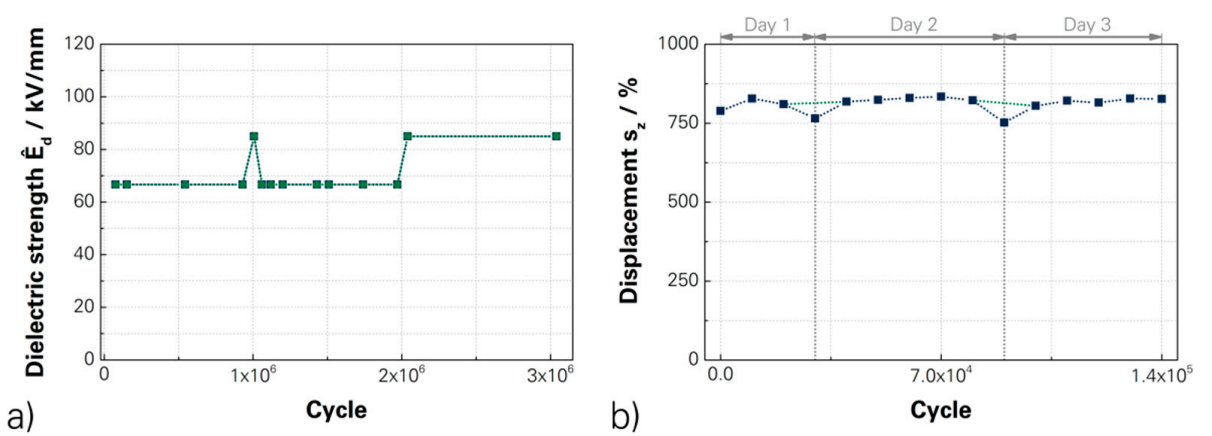

Figure 11. Investigated durability of the fully polymer DEAs for a (a) mechanical stress and cyclic measurement [15]; and (b) electro-mechanical stress under actuation conditions [15]—Table S1: actuators 10 and 11 .

\section{Conclusions and Outlook}

The investigations presented herein show that the newest DEAs have promising actuation properties, which follow the theoretical laws of DEAs. Additionally, full polymer actuators have been proven to have outstanding durability and interconnection between the assembled layers, which is a direct consequence of their monolithic structure. The entire development process for the associated material and technology was successfully completed and the processability and reproducibility were proven. The adjustment of the materials and layers allowed a reproduction of DEAs suitable for further application. The full polymer actuator achieved actuation displacements above $20 \%$ regarding thickness and exceeded more than 3 million cycles of mechanical stress without any malfunction or degradation of the DEA. The layers are not fragile and cracked after stress because the used modified silicone is able to resist elongations up to $75 \%$ and more.

Supplementary Materials: The used supplements following are available online at www.mdpi.com/2072-666X/ 7/10/172. Video S1: The characterization for large-scale DEAs.

Acknowledgments: This work was funded by Federal Ministry of Education and Research (BMBF) under grant 13 N. 10660. The project BMBF “Composite based new dielectric elastomer actuators (Candela)" was subdivided into four groups. The Fraunhofer-Institut für Werkstoff- und Strahltechnik, Dresden, Fraunhofer-Institut für Keramische Technologien und Systeme, Dresden, Chair of Polymeric Microsystems and Chair of Laser and Surface Technology, both located at the TU Dresden, investigated the full polymer actuator. The collaboration of the four groups enabled the success of the investigations and everyone applies many thanks. 
Author Contributions: O.J. mainly coined the idea of the Full Polymer Dielectric Elastomeric Actuators. T.K., R.L., G.P. and A.R. mainly conceived, designed and conducted the experiments, analysed the data and optimised the material, technology and characterisation. R.L., G.P. and A.R. conceived, designed and realised the high-precision experimental rig for characterisation of the actuation displacement. I.J. and T.K. conceived, conducted and optimised the modification process, the characterisation of the different materials and the production technology. A.S. produced and delivered the dielectric fillers and supported the investigations of the PMN-PT modified polydimethylsiloxane just as the characterisation of the actuator properties. O.J. produced and delivered the single-walled carbon nanotubes and supported the investigations of the electrical modified polydimethylsiloxane. T.K. wrote the manuscript and I.J., G.P. and A.R. supported the processing. E.B., I.J., O.J., A.R. and A.S. supervised and supported the project and investigations.

Conflicts of Interest: The authors declare no conflict of interest.

\section{References}

1. Röntgen, W. Ueber die durch Electricität bewirkten Form-und Volumenänderungen von dielectrischen Körpern. Ann. Phys. Chem. 1880, 247, 771-786. [CrossRef]

2. Pelrine, R.; Eckerle, J.; Chiba, S. Review of Artificial Muscle Approaches. In Proceedings of the International Symposium on Micro Machine and Human Science, Nagoya, Japan, 14-16 October 1992.

3. Pelrine, R.; Kornbluh, R.; Joseph, J.; Chiba, S. Electrostriction of Polymer Films for Microactuators. In Proceedings of the Tenth Annual International Workshop on Micro Electro Mechanical Systems, Nagoya, Japan, 26-30 January 1997; pp. 238-243.

4. Pelrine, R.; Kornbluh, R.; Joseph, J. Electrostriction of polymer dielectrics with compliant electrodes as a means of actuation. Sens. Actuators A 1998, 64, 77-85. [CrossRef]

5. Kornbluh, R.; Pelrine, R.; Eckerle, J.; Joseph, J. Electrostrictive Polymer Artificial Muscle Actuators. In Proceedings of the International Conference on Robotics and Automation, Leuven, Belgium, 16-20 May 998; pp. 2147-2154.

6. Brochu, P.; Pei, Q. Advances in Dielectric Elastomers for Actuators and Artificial Muscles. Macromol. Rapid Commun. 2010, 31, 10-36. [CrossRef] [PubMed]

7. Carpi, F. Electromechanically Active Polymers. Polym. Int. 2010, 59, 277-278. [CrossRef]

8. Brochu, P.; Niu, X.; Pei, Q. Acrylic interpenetrating polymer network dielectric elastomers for energy harvesting. In Proceedings of the Conference Electroactive Polymer Actuators and Devices, San Diego, CA, USA, 9-10 March 2010.

9. Pugal, D.; Jung, K.; Aabloo, A.; Kim, K.J. Ionic polymer-metal composite mechanoelectrical transduction: Review and perspectives. Polym. Int. 2010, 59, 279-289. [CrossRef]

10. Carpi, F.; Kornbluh, R.; Sommer-Larsen, P.; Alici, G. Electroactive polymer actuators as artificial muscles: Are they ready for bioinspired applications? Bioinspir. Biomim. 2011, 6, 045006. [CrossRef] [PubMed]

11. Bauer, S.; Keplinger, C. Dielectric-Elastomer Actuators Deliver Clean Energy; SPIE Newsroom: Bellingham, WA, USA, 2011.

12. Grauer, M.; Denes, I.; Köllnberger, A.; Kovacs, G. EpoSil—Gaining Sea Power with EAP. In Proceedings of the International Conference on New Actuators, Bremen, Germany, 18-20 June 2012; pp. 391-393.

13. Maiolino, P.; Galantini, F.; Mastrogiovanni, F.; Gallone, G.; Cannata, G.; Carpi, F. Soft dielectrics for capacitive sensing in robot skins: Performance of different elastomer types. Sens. Actuators A 2015, 226, 37-47. [CrossRef]

14. Jiang, L.; Betts, A.; Kennedy, D.; Jerrams, S. Improving the electromechanical performance of dielectric elastomers using silicone rubber and dopamine coated barium titanate. Mater. Des. 2015, 85, 733-742. [CrossRef]

15. Köckritz, T. Entwicklung Neuartiger Elektroaktiver Polymere auf Basis Vollpolymerer Monolithischer Schichtaufbauten. Ph.D. Thesis, TU Dresden, Dresden, Germany, February 2016.

16. Benslimane, M.; Gravesen, P.; Sommer-Larsen, P. Mechanical properties of Dielectric Elastomer Actuators with smart metallic compliant electrodes. In Proceedings of the Conference on Electro-Active Polymer Actuators and Devices, San Diego, CA, USA, 17-19 March 2002; pp. 150-157.

17. Bar-Cohen, Y. Electroactive polymer (EAP) Actuators as Artificial Muscles-Reality, Potential, and Challenges, 2nd ed.; SPIE Press: Bellingham, WA, USA, 2004.

18. Benslimane, M.; Kiil, H.-E.; Tryson, M. Dielectric electro-active polymer push actuators: Performance and challenges. Polym. Int. 2010, 59, 415-421. [CrossRef] 
19. Li, B.; Chen, H.; Qiang, J.; Hu, S.; Zhu, Z.; Wang, Y. Effect of mechanical pre-stretch on the stabilization of dielectric elastomer actuation. J. Phys. D Appl. Phys. 2011, 44, 155301. [CrossRef]

20. Mößinger, H.; Haus, H.; Schlaak, H. New Electrical Interconnection Techniques for Dielectric Elastomer Stack Transducers with Improved Lifetime. In Proceedings of the International Conference on New Actuators, Bremen, Germany, 18-20 June 2012; pp. 383-386.

21. Cakmak, E.; Fang, X.; Yildiz, O.; Bradford, P.D.; Ghosh, T.K. Carbon nanotube sheet electrodes for anisotropic actuation of dielectric elastomers. Carbon 2015, 89, 113-120. [CrossRef]

22. Matysek, M.; Lotz, P.; Schlaak, H. Lifetime investigation of dielectric elastomer stack actuators. IEEE Trans. Dielectr. Electr. Insul. 2011, 18, 89-96. [CrossRef]

23. Kiil, H.-E.; Benslimane, M. Scalable industrial manufacturing of DEAP. In Proceedings of the Conference on Electro-Active Polymer Actuators and Devices, San Diego, CA, USA, 9-10 March 2009.

24. Rødgaard, M. Piezoelectric Transformer Based Power Converters; Design and Control. Ph.D. Thesis, Technical University of Denmark, Lyngby, Denmark, September 2012.

25. Jost, O. Actuator Element and Use Thereof. Patent WO 2010020242 A3, 15 April 2010.

26. Dow Corning. Silicone Elastomer; Sylgard 184; Dow Corning: Midland, MI, USA, 2014.

27. Roch, A.; Jost, O.; Schultrich, B.; Beyer, E. High-yield synthesis of single-walled carbon nanotubes with a pulsed arc-discharge technique. Phys. Stat. Solidi B 2007, 244, 3907-3910. [CrossRef]

28. Roch, A.; Märcz, M.; Richter, U.; Leson, A.; Beyer, E.; Jost, O. Multi-component catalysts for the synthesis of SWCNT. Phys. Stat. Solidi B 2009, 246, 2511-2513. [CrossRef]

29. Roch, A.; Roch, T.; Talens, E.; Kaiser, B.; Lasagni, A.; Beyer, E.; Jost, O.; Cuniberti, G.; Leson, A. Selective laser treatment and laser patterning of metallic and semiconducting nanotubes in single walled carbon nanotube films. Diam. Relat. Mater. 2014, 45, 70-75. [CrossRef]

30. Gupta, S.; Bedekar, P.; Kulkarni, A. Synthesis, dielectric and microstructure studies of lead magnesium niobate stabilised using lead titanate. Ferroelectrics 1996, 189, 17-25. [CrossRef]

31. Schönecker, A.; Gebhardt, S. Oxide Targets for Integrated Thin Films. 2012. Available online: http://www. ikts.fraunhofer.de/en/communication/publications/annual_reports.html (accessed on 29 July 2016).

32. Tichy, J.; Erhart, J.; Kittinger, E.; Prívratská, J. Fundamentals of Piezoelectric Sensorics—Mechanical, Dielectric, and Thermodynamical Properties of Piezoelectric Materials; Springer: Berlin/Heidelberg, Germany, 2010.

33. Köckritz, T.; Jansen, I. Modification of polydimethylsiloxane based on the integration of carbon allotropes to achieve outstanding material properties for novel fields of application. Int. J. Adhes. Adhes. 2016. submitted.

34. Köckritz, T.; Wehnert, F.; Pap, J.-S.; Jansen, I. Increasing the Electrical Values of Polydimethylsiloxane by the Integration of Carbon Black and Carbon Nanotubes: A Comparison of the Effect of Different Nanoscale Fillers. J. Alloy. Compd. 2015, 51, 221-222.

35. Köckritz, T.; Wehnert, F.; Pap, J.-S.; Jansen, I. Comparison of different nanoscale fillers for electrical modification of silicone. In Proceedings of the International Nanotechnology Symposium, Dresden, Germany, 1-3 July 2014.

36. Köckritz, T.; Wehnert, F.; Pap, J.-S.; Jansen, I. Comparison of different nanoscale fillers according to their ability to change electrical and rheological values of adhesives. In Proceedings of the World Congress on Adhesion and Related Phenomena, Nara, Japan, 7-11 September 2014.

37. Pötschke, P.; Fornes, T.; Paul, D. Rheological behavior of multiwalled carbon nanotube/polycarbonate composites. Polymer 2002, 43, 3247-3255. [CrossRef]

38. Utracki, L. Flow and flow orientation of composites containing anisometric particles. Polym. Compos. 1986, 7, 274-282. [CrossRef]

39. Deutsches Institut für Normung. Electric Strength of Insulating Materials-Test Methods; DIN EN 60243-2; Beuth Verlag: Berlin, Germany, 2014.

40. The American Society for Testing Materials. Standard Test Methods for D-C Resistance or Conductance of Insulating Materials; ASTM D 257-99; ASTM International: West Conshohocken, PA, USA, 1998.

41. Lisowski, M.; Kacprzyk, R. Changes proposed for the IEC 60093 Standard concerning measurements of the volume and surface resistivities of electrical insulating materials. IEEE Trans. Dielectr. Electr. Insul. 2006, 13, 139-145. [CrossRef]

42. Keithley Instruments. Model 6517B Electrometer-User's Manual. 2010. Available online: http://www. tequipment.net/Keithley6517B.html?search=true (accessed on 23 March 2014). 
43. Keithley Instruments. Model 8009 Resistivity Test Fixture-Instruction Manual. 2010. Available online: http:/ / www.tequipment.net/Keithley8009.html?search=true (accessed on 23 March 2014).

44. Deutsches Institut für Normung. Plastics-Measurement of Resistivity of Conductive Plastics; DIN EN ISO 3915; Beuth Verlag: Berlin, Germany, 1999.

45. Smits, F. Measurement of sheet resistivities with the four-point probe. Bell Labs Tech. J. 1958, 37, 711-718. [CrossRef]

46. The American Society for Testing Materials. Standard Test Method for D-C Resistance or Conductance of Moderately Conductive Materials; ASTM D 4496-87; ASTM International: West Conshohocken, PA, USA, 1998.

47. Keithley Instruments. Multimeter 2000-User Manual. 2013. Available online: http://www.tequipment. net/Keithley2000-20.html?search=true\#tab-documents (accessed on 27 November 2013).

48. Deutsches Institut für Normung. Plastics-Determination of Tensile Properties; DIN EN ISO 527-1; Beuth Verlag: Berlin, Germany, 2012.

49. Deutsches Institut für Normung. Plastics-Determination of Tensile Properties; DIN EN ISO 527-3; Beuth Verlag: Berlin, Germany, 2003.

50. American Society for Testing Materials. Standard Test Method for Tensile Properties of Plastics; ASTM D 638-14; ASTM International: West Conshohocken, PA, USA, 2014.

51. Deutsches Institut für Normung. Adhesives-T-Peel Test for Flexible-to-Flexible Bonded Assemblies; DIN EN ISO 11339; Beuth Verlag: Berlin, Germany, 2010.

52. American Society for Testing Materials. Standard Test Method for Peel Resistance of Adhesives (T-Peel Test); ASTM D 1876-01; ASTM International: West Conshohocken, PA, USA, 2001.

53. Wissler, M.; Mazza, E. Modeling of a pre-strained circular actuator made of dielectric elastomers. Sens. Actuators A 2005, 120, 184-192. [CrossRef]

54. Zygo Corp. ZMI 7702 Laser Head-Technical Datasheet. 2009. Available online: http://www.zygo.com/?/ met/markets/stageposition/zmi/laserheads/ (accessed on 11 May 2015).

55. Hameg Instruments. Hameg HM8142-Power Supplies. 2014. Available online: http://www.helmut-singer. de/stock/-1672028357.html (accessed on 4 September 2015).

56. Trek Inc. Trek Model 609B-3 - High-Voltage Power Amplifier. 2013. Available online: http://www.trekinc. com/products/609B-3.asp (accessed on 4 September 2015).

57. Kofod, G.; Sommer-Larsen, P. Silicone dielectric elastomer actuators: Finite-elasticity model of actuation. Sens. Actuators A 2005, 122, 273-283. [CrossRef]

58. Lotz, P. Dielektrische Elastomerstapelaktoren für ein Peristaltisches Fluidfördersystem. Ph.D. Thesis, Technischen Universität Darmstadt, Darmstadt, Germany, November 2009.

59. Lotz, P.; Matysek, M.; Schlaak, H. Fabrication and application of miniaturized dielectric elastomer stack actuators. IEEE/ASME Trans. Mechatron. 2011, 16, 58-66. [CrossRef]

60. Min, C.; Shen, X.; Shi, Z.; Chen, L.; Xu, Z. The electrical properties and conducting mechanisms of carbon nanotube/polymer nanocomposites: A review. Polym. Plast. Technol. Eng. 2010, 49, 1172-1181. [CrossRef]

61. Aguilar, J. Influence of carbon nanotube clustering on the electrical conductivity of polymer composite films. Express Polym. Lett. 2010, 4, 292-299. [CrossRef]

62. Mullins, L. Softening of Rubber by Deformation. Rubber Chem. Technol. 1969, 42, 339-362. [CrossRef]

63. Risse, S.; Kussmaul, B.; Krüger, H.; Waché, R.; Kofod, G. DEA material enhancement with dipole grafted PDMS networks. In Proceedings of the Conference on Electroactive Polymer Actuators Devices, San Diego, CA, USA, 8-9 March 2011.

64. Diaz, R.; Diani, J.; Gilormini, P. Physical interpretation of the Mullins softening in a carbon-black filled SBR. Polymer 2014, 55, 4942-4947. [CrossRef]

(C) 2016 by the authors; licensee MDPI, Basel, Switzerland. This article is an open access article distributed under the terms and conditions of the Creative Commons Attribution (CC-BY) license (http://creativecommons.org/licenses/by/4.0/). 